\begin{tabular}{|c|}
\hline $\begin{array}{r}\text { PRAMANA } \\
\text { - journal of } \\
\text { physics }\end{array}$ \\
\hline
\end{tabular}

\title{
Erratum to: Allowable irreducible representations of the point groups with five-fold rotational axes ${ }^{\dagger}$
}

\author{
K RAMA MOHANA RAO ${ }^{1}$, B SIMHACHALAM ${ }^{2, *}$ and P HEMAGIRI RAO ${ }^{3}$ \\ ${ }^{1}$ MCA Department, Kaushik College of Engineering, Visakhapatnam 531 163, India \\ ${ }^{2}$ Department of Mathematics, GITAM University, Visakhapatnam 530 045, India \\ ${ }^{3}$ MCA Department, Vasavi College of Engineering, Hyderabad 500 031, India \\ *Corresponding author. E-mail: simhachalam1983@gmail.com
}

MS received 5 August 2011; accepted 18 April 2012

\begin{abstract}
Allowable irreducible representations of the point groups with five-fold rotations - that represent the symmetry of the quasicrystals in two and three dimensions - are derived by employing the little group technique in conjunction with the solvability property. The point groups $D_{5 h}(\overline{10} \mathrm{~m} 2)$ and $I_{h}\left(\frac{2}{m} \overline{3} \overline{5}\right)$ are taken to illustrate the method.
\end{abstract}

Keywords. Allowable irreducible representations of little groups; solvability property; composition series; induced and engendered representations.

PACS No. 02.20. - a

\section{Introduction}

Character table for the 32 crystallographic point groups - that represent the point symmetry of the crystals and the symmetry of some molecules - as also for the nine non-crystallographic point groups with five-fold rotation that represent the symmetry of quasicrystals in two and three dimensions - along with the method for obtaining them are available in literature [1-3]. Out of the well-known methods employed to find the irreducible representations (IRs) of finite groups, the application of the little group method in conjunction with the solvability property [4] is considered as the most elegant and simple method. This classic method explained in Bradley [5] and Bradley and Cracknell [3] was applied to the plane group $P 4 g$ and to the space groups by Raghavacharyulu [6] and to the crystallographic point groups by Ramachandra Rao [7]. A slightly different approach to this little group method described by Bhagavantam and Venkatarayudu [8], was applied by Krishnamurthy et al [9] to obtain all the one-dimensional allowable irreducible representations (AIRs) of the appropriate little groups that induce the degenerate representations of the 32 crystallographic point groups.

\footnotetext{
${ }^{\dagger}$ The original article published online with DOI:10.1007/s12043-012-0334-1 contained some errors. The corrected article is re-published here in its entirety.
} 


\section{K Rama Mohana Rao, B Simhachalam and P Hemagiri Rao}

Among the finite groups with five-fold rotational symmetry, the icosahedral point group $I(235)$ is the most complex point group which has been the subject of interest for several group - theoretical physicists. The discovery of quasicrystals [10] has revived the interest of these researchers to study the seven pentagonal point groups $C_{5}(5), S_{10}(\overline{5}), C_{5 h}(\overline{10})$, $D_{5}(52), D_{5 h}(\overline{10} m 2), C_{5 v}(5 m), D_{5 d}(\overline{5} 2 m)$ and the two icosahedral point groups $I(235)$ and $I_{h}\left(\frac{2}{m} \overline{3} \overline{5}\right)$ that exhibit five-fold rotational symmetry. The IRs of the icosahedral point groups have been studied by Backhouse and Gard [11]. Using a new approach to the group representation theory - which in turn paved way for eigenfunction method - Chen [12] obtained the characters, IRs and isoscalar factors for the icosahedral point groups which have been of interest in connection with the vibrational and electronic propagation $[13,14]$. Interest in the icosahedral point groups also stemmed from the icosahedral symmetry of biological macromolecules [15].

In this note, an attempt has been made to obtain/derive the allowable irreducible representations (AIRs) of the little groups that induce various IRs of the seven pentagonal point groups and the icosahedral point group $I_{h}\left(\frac{2}{m} \overline{3} \overline{5}\right)$ by applying the little group method in conjunction with the solvability property. The application of this powerful technique is illustrated with the help of two composition series:

(i) $D_{5 h}(\overline{10} m 2) \supset D_{5}(52) \supset C_{5}(5) \supset C_{1}(1)$

(ii) $I_{h}\left(\frac{2}{m} \overline{35}\right) \supset I(235)$.

In $\S 2$, we shall first familiarize the reader with the necessary basic terminology of the method and illustrate each one of them with an example from the chosen series. To aid the discussion, we shall refer to the character table of the point groups $D_{5 h}(\overline{10} m 2), D_{5}(52)$, $C_{5}(5), I_{h}\left(\frac{2}{m} \overline{35}\right)$ and $I(235)$ provided in $\S 2$ and 3 and to the factor groups involving these groups in the considered composition series. The generating elements and the defining relations are listed for each group in the considered series along with the character table and the multiplication tables for the elements of these groups are available in [16].

\section{Some definitions and basic terminology}

In the discussion that follows, $G$ is a finite group and $H$ is a normal subgroup of $G$. We say that $G$ is solvable if the order of the factor groups $H_{i} / H_{i+1}$ in the composition series $G=H_{0} \supset \ldots \supset H_{i} \supset H_{i+1} \supset \ldots \supset C_{1}=E$ are prime numbers. For example, the group $D_{5 h}$ is solvable since the composition indices 5, 2, 2 in the composition series $D_{5 h} \supset D_{5} \supset C_{5} \supset C_{1}(=E)$ are prime numbers.

(i) Conjugate representation $\Delta^{A}$ : A representation $\Delta^{A}$ of $H$ conjugate to $\Delta$ relative to $G$ is defined by $\Delta^{A} \rightarrow D^{\Delta}\left(A H A^{-1}\right)$ where $D^{\Delta}\left(A H A^{-1}\right)$ is the matrix representing the element $A$ in the representation $\Delta$. If $\Delta$ and $\Delta^{A}$ are equivalent, then $\Delta$ is called self-conjugate.

In the group $C_{5}$ the total symmetric representation $A$ is self-conjugate whereas the pair of 1-d complex representations $E_{a}$ and $E_{b}$ are conjugate but inequivalent to each other, relative to the group $D_{5}$. 
(ii) Little groups of the second kind $L^{(2)}$ and of the first kind $L^{(1)}$ : All the elements of a group $G$ for which $\Delta$ is self-conjugate, i.e., $\Delta \cdot \equiv \cdot \Delta^{A}$ form the little group of the second kind (or little group) relative to $(G, H, \Delta)$ and is denoted by $L^{(2)}(G, H, \Delta)$. The quotient group $L^{(2)} / H$ is the corresponding little group of the first kind (or little co-group) and is denoted by $L^{(1)}(G, H, \Delta)$.

If $G=D_{5}$ and $H=C_{5}$, then for the IR $A$ of $C_{5}, L^{(2)}(G, H, \Delta)=D_{5}$ and $L^{(1)}(G, H$, $\Delta)=D_{5} / C_{5} \cong C_{2}$.

(iii) Orbit (or Star) $\theta$ : An orbit $\theta$ of the normal subgroup $H$ of $G$ is the set of all inequivalent IRs of $H$ which are mutually conjugate relative to the elements of $G$. The number of IRs in $\theta$ gives the order of the orbit $\theta$.

The group $C_{5}$ has three orbits: $\theta_{1}=\{A\}, \theta_{2}=\left\{E_{a_{1}}, E_{a_{2}}\right\}, \theta_{3}=\left\{E_{b_{1}}, E_{b_{2}}\right\}$ with respect to $D_{5}$. Similarly, $D_{5}$ has four orbits: $\theta_{1}=\left\{A_{1}\right\}, \theta_{2}=\left\{A_{2}\right\}, \theta_{3}=\left\{E_{1}\right\}, \theta_{4}=\left\{E_{2}\right\}$ with respect to $D_{5 h}$.

(iv) Subduced representation $\left(\Gamma^{S}=\Gamma \downarrow H\right)$ : Let $D^{\Gamma}(A)$ and $D^{\Gamma}(B)$ be the matrices representing the elements $A$ and $B$ in the representation $\Gamma$ of $G$. In the representation $\Gamma \rightarrow D^{\Gamma}(A)$ of $G$, the matrices $D^{\Gamma}(B)$ which are images of $H$, form a representation of $H$ called the subduced representation $\Gamma^{S}$ of $H$ and is denoted by $\Gamma \downarrow H=\Gamma^{S} \rightarrow D^{\Gamma}(B)$. Here $\Gamma^{S}$ will be of the same dimension as $\Gamma$ and is in general reducible.

If $G=D_{5}$ and $H=C_{5}$, then $A_{1} \downarrow C_{5}=A\left(C_{5}\right), A_{2} \downarrow C_{5}=A\left(C_{5}\right), E_{1} \downarrow C_{5}=E_{a}\left(C_{5}\right)$ and $E_{2} \downarrow C_{5}=E_{b}\left(C_{5}\right)$. Here $A_{1}, A_{2}, E_{1}, E_{2}$ are the IRs of $D_{5}$ (table 1) and $A, E_{a}, E_{b}$ are the IRs of $C_{5}$ (table 2).

Table 1. Character table for the point group $D_{5}$.

\begin{tabular}{ccccc}
\hline$D_{5}$ & $E$ & $2 C_{5}$ & $2 C_{5}^{2}$ & $5 C_{2}$ \\
\hline$A_{1}$ & 1 & 1 & 1 & 1 \\
$A_{2}$ & 1 & 1 & 1 & 1 \\
$E_{1}$ & 2 & $2 \cos 72^{\circ}$ & $2 \cos 144^{\circ}$ & 0 \\
$E_{2}$ & 2 & $2 \cos 144^{\circ}$ & $2 \cos 72^{\circ}$ & 0 \\
\hline
\end{tabular}

Generating elements: $C_{5}, C_{2}$; Defining relations: $\left(C_{5}\right)^{5}=\left(C_{2}\right)^{2}=E$ and $C_{2} C_{5}=C_{2}^{\prime}$.

$$
\begin{array}{ccc}
\text { IR of } D_{5} & C_{5} & C_{2} \\
E_{1} & \left(\begin{array}{cc}
\omega & 0 \\
0 & \omega^{4}
\end{array}\right) & \left(\begin{array}{ll}
0 & 1 \\
1 & 0
\end{array}\right) \\
E_{2} & \left(\begin{array}{cc}
\omega^{2} & 0 \\
0 & \omega^{3}
\end{array}\right) & \left(\begin{array}{ll}
0 & 1 \\
1 & 0
\end{array}\right)
\end{array}
$$




\section{K Rama Mohana Rao, B Simhachalam and P Hemagiri Rao}

Table 2. Character table for the point group $C_{5}$.

\begin{tabular}{|c|c|c|c|c|c|c|}
\hline$C_{5}$ & & $E$ & $C_{5}$ & $C_{5}^{2}$ & $C_{5}^{3}$ & $C_{5}^{4}$ \\
\hline$A$ & & 1 & 1 & 1 & 1 & 1 \\
\hline$E_{a}$ & $\begin{array}{l}E_{a_{1}} \\
E_{a_{2}}\end{array}$ & 1 & $\omega$ & $\omega^{2}$ & $\omega^{3}$ & $\omega^{4}$ \\
\hline \multirow{3}{*}{$E_{b}$} & \multirow{3}{*}{$\begin{array}{l}E_{b_{1}} \\
E_{b_{2}}\end{array}$} & 1 & $\omega^{4}$ & $\omega^{3}$ & $\omega^{2}$ & $\omega$ \\
\hline & & 1 & $\omega^{2}$ & $\omega^{4}$ & $\omega$ & $\omega^{3}$ \\
\hline & & 1 & $\omega^{3}$ & $\omega$ & $\omega^{4}$ & $\omega^{2}$ \\
\hline
\end{tabular}

Generating element: $C_{5}$; Defining relations: $\left(C_{5}\right)^{5}=E$.

(v) Induced representation $\left(\Gamma \equiv \Delta^{\alpha}=\Delta \uparrow G\right)$ : Let $|G|=g,|H|=h$ and $G=\sum_{i=1}^{g / h} A_{i} H$ be a left coset decomposition of $G$ relative to $H$. Define $\sigma(A, B)$ as a matrix of dimension $g / h$ having the elements

$$
\sigma_{i, j}(A, B)=\left\{\begin{array}{l}
1, \text { if } A_{i} B A_{j}^{-1}=A \\
0, \quad \text { otherwise }
\end{array} .\right.
$$

If $D^{\Gamma}(A)$ is the matrix representing the element $A$ in the IR $\Gamma$ of $G$ and $D^{\Delta}(B)$ is the matrix representing the element $B$ in the IR $\Delta$ of $H$, then

$$
D^{\Gamma}(A)=\sum_{B \in H} \sigma(A, B) \otimes D^{\Delta}(B) .
$$

Then $\Gamma \rightarrow D^{\Gamma}(A)$ is a representation of $G$ of dimension $(g / h) d$, induced by the IR $\Delta$ of $H$ and is denoted by $\Gamma=\Delta \uparrow G$.

Let $G=D_{5}$ and $H=C_{5}, D_{5}=E C_{5} \cup C_{2} C_{5}$ and $E_{1}$ be an IR of $D_{5}, E_{a_{1}}$ be an IR of $C_{5}$. Then

$$
\begin{aligned}
D^{E_{1}}\left(C_{5}\right)= & \sigma\left(C_{5}, E\right) \otimes D^{E_{a_{1}}}(E)+\sigma\left(C_{5}, C_{5}\right) \otimes D^{E_{a_{1}}}\left(C_{5}\right)+\sigma\left(C_{5}, C_{5}^{2}\right) \otimes D^{E_{a_{1}}}\left(C_{5}^{2}\right) \\
& +\sigma\left(C_{5}, C_{5}^{3}\right) \otimes D^{E_{a_{1}}}\left(C_{5}^{3}\right)+\sigma\left(C_{5}, C_{5}^{4}\right) \otimes D^{E_{a_{1}}}\left(C_{5}^{4}\right) \\
= & \left(\begin{array}{cc}
\omega & 0 \\
0 & \omega^{4}
\end{array}\right),
\end{aligned}
$$

where $\omega=\exp (2 \pi i / 5)$.

Similarly,

$$
\begin{aligned}
D^{E_{1}}\left(C_{2}\right)= & \sigma\left(C_{2}, E\right) \otimes D^{E_{a_{1}}}(E)+\sigma\left(C_{2}, C_{5}\right) \otimes D^{E_{a_{1}}}\left(C_{5}\right)+\sigma\left(C_{2}, C_{5}^{2}\right) \otimes D^{E_{a_{1}}}\left(C_{5}^{2}\right) \\
& +\sigma\left(C_{2}, C_{5}^{3}\right) \otimes D^{E_{a_{1}}}\left(C_{5}^{3}\right)+\sigma\left(C_{2}, C_{5}^{4}\right) \otimes D^{E_{a_{1}}}\left(C_{5}^{4}\right) \\
= & \left(\begin{array}{ll}
0 & 1 \\
1 & 0
\end{array}\right) .
\end{aligned}
$$




\section{Allowable irreducible representations of the point groups}

Hence the IR $E_{1}$ of $D_{5}$ is induced by $E_{a_{1}}$ of $C_{5}$, i.e. $E_{a_{1}} \uparrow D_{5}$. We observe here that the actual form of the induced IR depends on the choice of the coset representative $A_{i}$. However, an alternative choice of $A_{i}$ may yield an equivalent IR.

(vi) Engendered representation: A representation of the group $G$ obtained from a representation of $G / H$ is called an engendered representation of $G$. If a representation of $G / H$ is irreducible, then the engendered representation of $G$ is also irreducible.

Consider $D_{5} / C_{5} \cong C_{2}$. It can be seen that the total symmetric IR $A_{1}$ of $C_{2}$ engender the total symmetric IR $A_{1}$ of $D_{5}$ and the 1-d alternating IR of $C_{2}$ engender the alternating IR $A_{2}$ of $D_{5}$.

(vii) Allowable irreducible representation (AIR): $\gamma$ is said to be an AIR of the little group $L^{(2)}(G, H, \Delta)$ if $\gamma$ subduces an integer multiple $m$ of $\Delta$ on $H$, i.e. $\gamma \downarrow H=m \Delta$.

The 1-d IR $A_{1}$ and $A_{2}$ of $D_{5}$ are the AIRs of $L^{(2)}\left(D_{5}, C_{5}, A\right)=D_{5}$.

\section{The little group method}

The little group method of finding the IRs of a finite group $G$ from those of the IRs of its maximal normal subgroup $H$ in a chosen composition series in conjunction with solvability property involves the following basic steps:

(a) Express the group $G$ in terms of the composition series $G=H_{0} \supset H_{1} \supset \cdots \supset$ $H_{i} \supset H_{i+1} \supset \cdots \supset C_{1}=E$. Since $G$ is solvable, the quotient group $G / H$ is cyclic and is of prime order $\left(g / h_{i}\right)=\alpha_{i}$ (say) for $H=H_{i}$ and the IRs for $H$ are supposed to be known.

(b) Classify the IRs of $H$ into orbits $\theta_{i}$ with respect to $G$. The order of an orbit $\theta_{i}$ is either $\alpha_{i}$ or 1 .

(c) Choose an IR, say $\Delta_{i}$ of dimension $d_{i}$ from each orbit $\theta_{i}$. Then, (i) if the order of $\theta_{i}$ is $\alpha_{i}, L^{(2)}\left(G, H, \Delta_{i}\right)=H$ and there is a unique IR of dimension $(g / h) d_{i}$ of $G$ obtained by inducing with $\Delta_{i}$. (ii) If the order of $\theta_{i}$ is 1 and $\Delta_{i}$ is non-degenerate, then $\alpha_{i}$ IRs of $G$ are engendered by the IRs of $G / H$. If $\Delta_{i}$ is degenerate, then IRs of $G$ are obtained from those of the IRs of $G / H$ with the help of the defining relations for the generators of $G$.

(d) If $\gamma$ is an AIR of $L^{(2)}(G, H, \Delta)$, then $\Gamma=\gamma \uparrow G$ is irreducible. If the AIRs of only one little group per orbit $\theta$ of $H$ are used to induce the IRs of $G$, then each one of the IR of $G$ occurs only once.

It can be seen (table 3 ) that composition series exist among the seven pentagonal point groups which are subgroups of either of the groups $D_{5 h}(\overline{10} \mathrm{~m} 2)$ or $D_{5 d}(\overline{5} 2 \mathrm{~m})$ and the icosahedral point group $I(235)$ is a normal subgroup of $I_{h}\left(\frac{2}{m} \overline{3} \overline{5}\right)$. Furthermore, the point group $I(235)$ is not solvable, in that, it has no non-trivial normal subgroup. Taking the composition series for the group $D_{5 h}(\overline{10} m 2): D_{5 h} \supset D_{5} \supset C_{5} \supset C_{1}(=E)$ we observe that $C_{5} / C_{1} \equiv C_{5}$ is a cyclic group of order 5 . The point group $C_{5}$ has three orbits $\theta_{1}=\{A\}$, $\theta_{2}=\left\{E_{a_{1}}, E_{a_{2}}\right\}, \theta_{3}=\left\{E_{b_{1}}, E_{b_{2}}\right\}$ with respect to $D_{5}$ and $L^{(2)}\left(D_{5}, C_{5}, A\right)=D_{5}$. The IR $A$ of $C_{5}$ engender the two IRs $A_{1}$ and $A_{2}$ of $D_{5}$. 
To find the $2 \times 2$ matrices $\sigma(A, B)$, we consider

$$
\sigma_{i, j}(A, B)=\left\{\begin{array}{l}
1, \text { if } A_{i} B \mathrm{~A}_{j}^{-1}=A \\
0, \quad \text { otherwise }
\end{array} .\right.
$$

For $A \in\left\{C_{2}, C_{5}\right\}$ and $B \in\left\{E, C_{5}, C_{5}^{2}, C_{5}^{3}, C_{5}^{4}\right\}$. Since $D^{\Gamma}(A)=\sum_{B \in H} \sigma(A, B) \otimes D^{\Delta}(B)$, for the IR $E_{1}$ of $D_{5}$ and for $A=C_{5}, A=C_{2}$, we get

$$
D^{E_{1}}\left(C_{5}\right)=\left(\begin{array}{cc}
\omega & 0 \\
0 & \omega^{4}
\end{array}\right), \quad D^{E_{1}}\left(C_{2}\right)=\left(\begin{array}{ll}
0 & 1 \\
1 & 0
\end{array}\right) .
$$

Similarly, for IR $E_{2}$ of $D_{5}$ and for $A=C_{5}, A=C_{2}$, we get respectively

$$
D^{E_{2}}\left(C_{5}\right)=\left(\begin{array}{cc}
\omega^{2} & 0 \\
0 & \omega^{3}
\end{array}\right), \quad D^{E_{2}}\left(C_{2}\right)=\left(\begin{array}{ll}
0 & 1 \\
1 & 0
\end{array}\right) .
$$

The matrices for the remaining elements of $D_{5}$ in the considered IR can be obtained using the generating relations $\left(C_{5}\right)^{5}=\left(C_{2}\right)^{2}=E$ and $C_{2} C_{5}=C_{2}^{\prime}$. The pair of 1-d complex IR $E_{a_{1}}, E_{a_{2}}$ induce the IR $E_{1}$ and $E_{b_{1}}, E_{b_{2}}$ induce the IR $E_{2}$ of $D_{5}$. The IRs $A_{1}$ and $A_{2}$ are two AIRs of $L^{(2)}\left(D_{5}, C_{5}, A\right)=D_{5}$. These results are shown in table 3 .

To obtain the AIRs of $D_{5}$ that induce the IRs of $D_{5 h}$, the IRs of $D_{5}$ are classified into four orbits $\theta_{1}=\left\{A_{1}\right\}, \theta_{2}=\left\{A_{2}\right\}, \theta_{3}=\left\{E_{1}\right\}, \theta_{4}=\left\{E_{2}\right\}$ relative to $D_{5 h}$. Since

Table 3. Schematic diagram showing the AIRs of the little group that induce the IRs of the point groups in the composition series $D_{5 h} \supset D_{5} \supset C_{5} \supset C_{1}(=E)$. The point groups within the parenthesis represent the little groups of the second kind $L^{(2)}(G, H, \Delta)$.

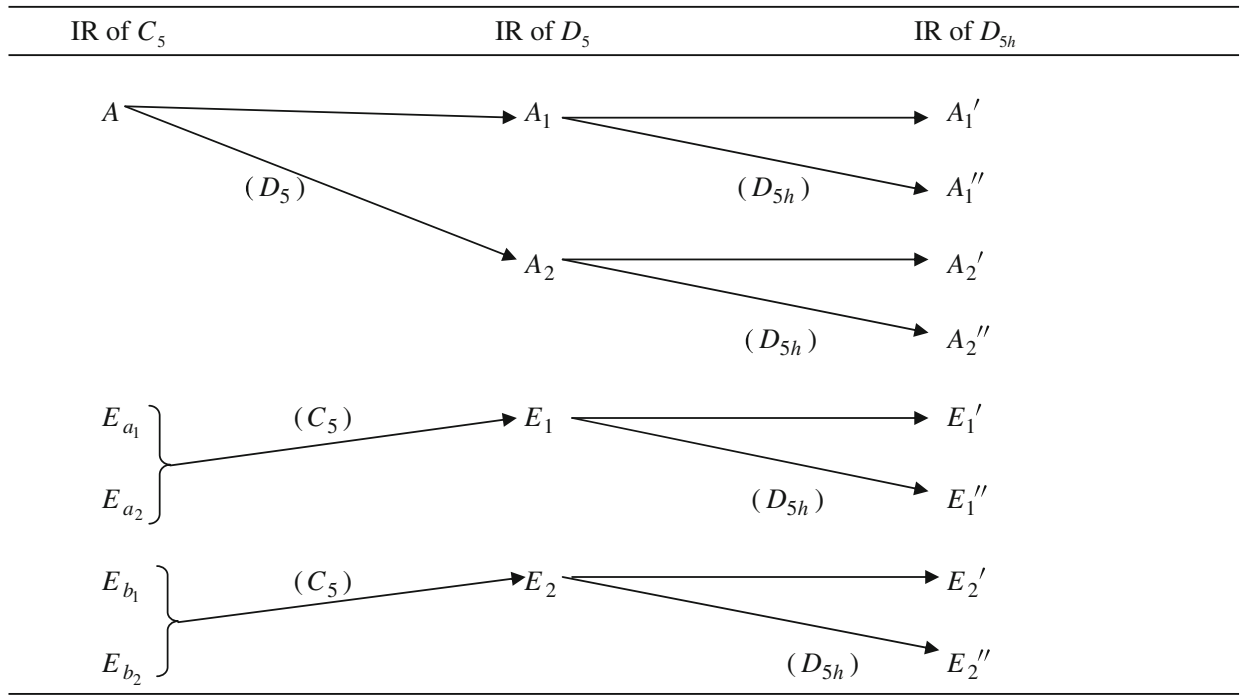


$D_{5 h} / D_{5} \cong C_{2}$, from the little group method all the IRs of $D_{5 h}$ are engendered from those of the IRs of $D_{5}$ : The IR $A_{1}$ of $D_{5}$ engenders $A_{1}^{\prime}, A_{1}^{\prime \prime}, A_{2}$ of $D_{5}$ engenders $A_{2}^{\prime}$, $A_{2}^{\prime \prime}, E_{1}$ of $D_{5}$ engenders $E_{1}^{\prime}, E_{1}^{\prime \prime}, E_{2}$ of $D_{5}$ engenders $E_{2}^{\prime}, E_{2}^{\prime \prime}$ of $D_{5 h}$. The matrices representing the generating elements $C_{5}, C_{2}, \sigma_{h}$ of $D_{5 h}$ for the IRs $E_{1}^{\prime}, E_{1}^{\prime \prime}, E_{2}^{\prime}, E_{2}^{\prime \prime}$ are provided in table 4 . The generating matrices for the remaining elements of $D_{5 h}$ in the considered IR can be obtained using the defining relations $\sigma_{h}^{2}=\sigma_{\vartheta}^{2}=C_{2}^{2}=C_{5}^{5}=E$ and $\sigma_{h} C_{2}=\sigma_{\vartheta}$. The results obtained for the considered composition series are shown in table 3 .

Following a similar procedure, the AIRs of the point groups that induce/engender the IRs of the other groups can be obtained, by a proper choice of the composition series.

In respect of the icosahedral point groups $I$ and $I_{h}$, since the group $I$ is not solvable, we start the little group method from the matrix representation of the generating elements $C_{2}$ and $C_{5}$ of $I$ corresponding to the IRs $F_{1}, F_{2}, G$ and $H$ as given by Matossi [17]. The IRs of $I$ are classified into five orbits $\theta_{1}=\{A\}, \theta_{2}=\left\{F_{1}\right\}, \theta_{3}=\left\{F_{2}\right\}, \theta_{4}=\{G\}$ and $\theta_{5}=\{H\}$, relative to $I_{h}$. Since $I_{h} / I \cong C_{2}$, which is of prime order, all the IRs of $I_{h}$ are engendered from those of the IRs of $I$ from the little group method, as shown in table 5 .

Table 4. Character table for the point group $D_{5 h}$.

\begin{tabular}{|c|c|c|c|c|c|c|c|c|}
\hline$D_{5 h}$ & $E$ & $2 C_{5}$ & $2 C_{5}^{2}$ & $5 C_{2}$ & $\sigma_{h}$ & $2 S_{5}$ & $2 S_{5}^{3}$ & $5 \sigma_{\vartheta}$ \\
\hline$A_{1}^{\prime}$ & 1 & 1 & 1 & 1 & 1 & 1 & 1 & 1 \\
\hline$A_{2}^{\prime}$ & 1 & 1 & 1 & -1 & 1 & 1 & 1 & -1 \\
\hline$E_{1}^{\prime}$ & 2 & $2 \cos 72^{\circ}$ & $2 \cos 144^{\circ}$ & 0 & 2 & $2 \cos 72^{\circ}$ & $2 \cos 144^{\circ}$ & 0 \\
\hline$E_{2}^{\prime}$ & 2 & $2 \cos 144^{\circ}$ & $2 \cos 72^{\circ}$ & 0 & 2 & $2 \cos 144^{\circ}$ & $2 \cos 72^{\circ}$ & 0 \\
\hline$A_{1}^{\prime \prime}$ & 1 & 1 & 1 & 1 & -1 & -1 & -1 & -1 \\
\hline$A_{2}^{\prime \prime}$ & 1 & 1 & 1 & -1 & -1 & -1 & -1 & 1 \\
\hline$E_{1}^{\prime \prime}$ & 2 & $2 \cos 72^{\circ}$ & $2 \cos 144^{\circ}$ & 0 & -2 & $-2 \cos 72^{\circ}$ & $-2 \cos 144^{\circ}$ & 0 \\
\hline$E_{2}^{\prime \prime}$ & 2 & $2 \cos 144^{\circ}$ & $2 \cos 72^{\circ}$ & 0 & -2 & $-2 \cos 144^{\circ}$ & $-2 \cos 72^{\circ}$ & 0 \\
\hline
\end{tabular}

Generating elements: $C_{5}, C_{2}, \sigma_{h}$; Defining relations: $\sigma_{h}^{2}=\sigma_{\vartheta}^{2}=C_{2}^{2}=C_{5}^{5}=E$ and $\sigma_{h} C_{2}=\sigma_{\vartheta}$.

$\begin{array}{cccc}\text { IR of } D_{5 h} & C_{5} & C_{2} & \sigma_{h} \\ E_{1}^{\prime} & \left(\begin{array}{cc}\omega & 0 \\ 0 & \omega^{4}\end{array}\right) & \left(\begin{array}{ll}0 & 1 \\ 1 & 0\end{array}\right) & \left(\begin{array}{ll}1 & 0 \\ 0 & 1\end{array}\right) \\ E_{2}^{\prime} & \left(\begin{array}{cc}\omega^{2} & 0 \\ 0 & \omega^{3}\end{array}\right) & \left(\begin{array}{ll}0 & 1 \\ 1 & 0\end{array}\right) & \left(\begin{array}{cc}1 & 0 \\ 0 & 1\end{array}\right) \\ E_{1}^{\prime \prime} & \left(\begin{array}{cc}\omega & 0 \\ 0 & \omega^{4}\end{array}\right) & \left(\begin{array}{ll}0 & 1 \\ 1 & 0\end{array}\right) & \left(\begin{array}{cc}-1 & 0 \\ 0 & -1\end{array}\right) \\ E_{2}^{\prime \prime} & \left(\begin{array}{cc}\omega^{2} & 0 \\ 0 & \omega^{3}\end{array}\right) & \left(\begin{array}{ll}0 & 1 \\ 1 & 0\end{array}\right) & \left(\begin{array}{cc}-1 & 0 \\ 0 & -1\end{array}\right)\end{array}$




\section{K Rama Mohana Rao, B Simhachalam and P Hemagiri Rao}

Table 5. Schematic diagram showing the IRs of the point group $I_{h}$ engendered from the IRs of the point group $I$. The point groups within parenthesis represent the little groups of the second kind $L^{(2)}(G, H, \Delta)$.

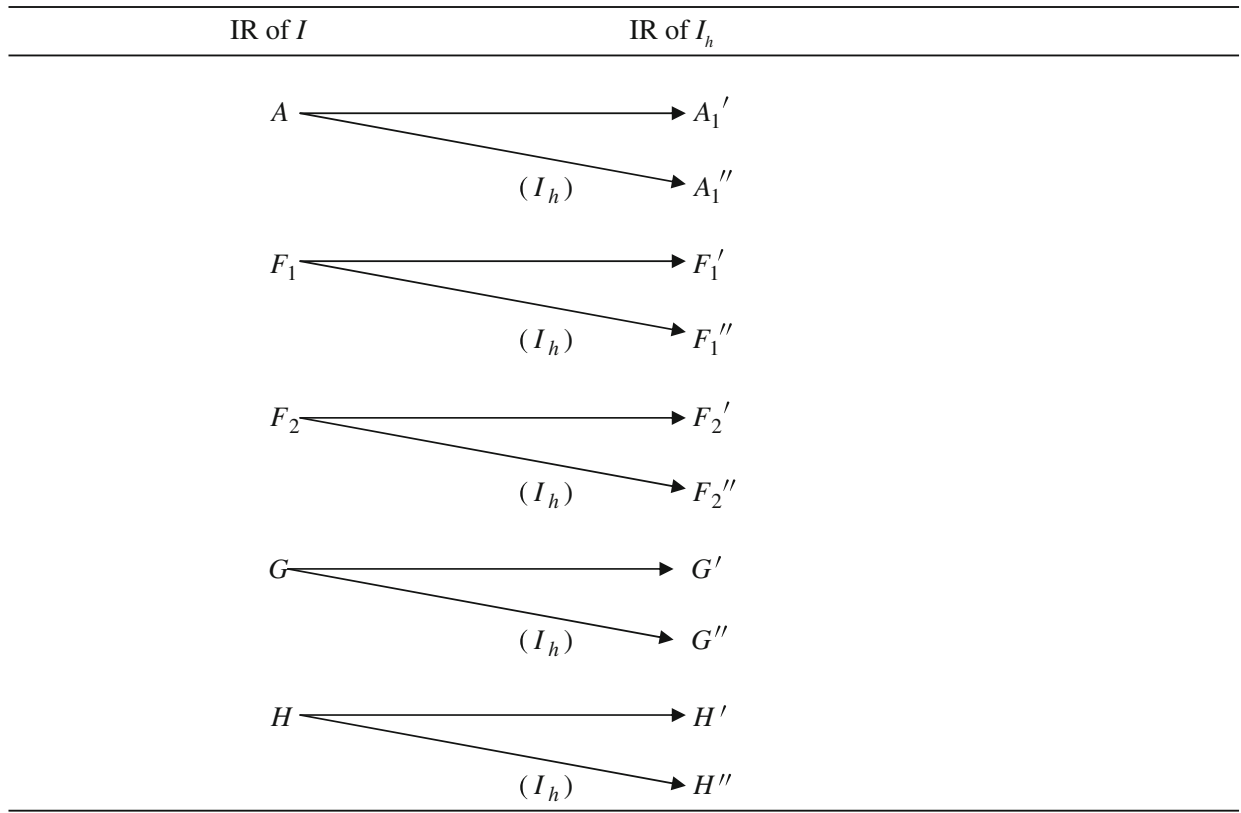

Defining relations of $I_{h}: i^{2}=\sigma^{2}=C_{2}^{2}=C_{5}^{5}=E, i C_{5}=S_{10}, i C_{3}=S_{6}, i C_{2}=\sigma$.

Matrix representation of the generating elements of the point group $I$ :

\begin{tabular}{|c|c|c|c|c|c|c|c|c|}
\hline \multirow{2}{*}{$\begin{array}{c}\text { IR of } I \\
F_{1}\end{array}$} & \multicolumn{3}{|c|}{$C_{5}$} & \multicolumn{5}{|c|}{$C_{2}$} \\
\hline & & $\left.\begin{array}{ccc}(\sqrt{5}-1) / 4 & -(\sqrt{\tau+2}) / 2 & 0 \\
(\sqrt{\tau+2}) / 2 & (\sqrt{5}-1) / 4 & 0 \\
0 & 0 & 1\end{array}\right)$ & & & $\left(\begin{array}{c}-1 \\
0 \\
0\end{array}\right.$ & $\begin{array}{c}0 \\
-1 / \sqrt{5} \\
-2 / \sqrt{5}\end{array}$ & $\left.\begin{array}{c}0 \\
-2 / \sqrt{5} \\
1 / \sqrt{5}\end{array}\right)$ & \\
\hline$F_{2}$ & ( & $\begin{array}{ccc}-(\sqrt{5}+1) / 4 & -(\sqrt{3-\tau}) / 2 & 0 \\
(\sqrt{3-\tau}) / 2 & -(\sqrt{5}+1) / 4 & 0 \\
0 & 0 & 1\end{array}$ & & & $\left(\begin{array}{c}-1 \\
0 \\
0\end{array}\right.$ & $\begin{array}{c}0 \\
1 / \sqrt{5} \\
2 / \sqrt{5}\end{array}$ & $\left.\begin{array}{c}0 \\
2 / \sqrt{5} \\
-1 / \sqrt{5}\end{array}\right)$ & \\
\hline$G$ & $\left(\begin{array}{c}(\sqrt{5}-1) / 4 \\
0 \\
0 \\
(\sqrt{\tau+2}) / 2\end{array}\right.$ & $\begin{array}{cc}0 & 0 \\
-(\sqrt{5}+1) / 4 & -(\sqrt{3-\tau}) / 2 \\
(\sqrt{3-\tau}) / 2 & -(\sqrt{5}+1) / 4 \\
0 & 0\end{array}$ & $\left.\begin{array}{c}-(\sqrt{\tau+2}) / 2 \\
0 \\
0 \\
(\sqrt{5}-1) / 4\end{array}\right)$ & & $\left(\begin{array}{r}-2 / \\
-1 / \\
0 \\
0\end{array}\right.$ & $\begin{array}{rr}\sqrt{5} & -1 / \\
\sqrt{5} & 2 / \sqrt{ } \\
& 0 \\
& 0\end{array}$ & $\left.\begin{array}{lll}\sqrt{5} & 0 & 0 \\
\sqrt{5} & 0 & 0 \\
0 & 0 & 1 \\
0 & 1 & 0\end{array}\right)$ & \\
\hline $\boldsymbol{H}$ & $\left(\begin{array}{cc}1 & 0 \\
0 & (\sqrt{5}-1) / 4 \\
0 & 0 \\
0 & 0 \\
0 & (\sqrt{\tau+2}) / 2\end{array}\right.$ & $\begin{array}{ccc}4 & 0 & 0 \\
& 0 & 0 \\
-(\sqrt{5}+1) / 4 & -(\sqrt{3-\tau}) / 2 \\
& (\sqrt{3-\tau}) / 2 & -(\sqrt{5}+1) / 4 \\
2 & 0 & 0\end{array}$ & $\begin{array}{c}0 \\
-(\sqrt{\tau+2}) / 2 \\
0 \\
0 \\
(\sqrt{5}-1) / 4\end{array}$ & $\left(\begin{array}{c}-1 / 5 \\
0 \\
0 \\
\sqrt{12} / 5 \\
\sqrt{12} / 5\end{array}\right.$ & $\begin{array}{c}0 \\
-1 / \sqrt{5} \\
2 / \sqrt{5} \\
0 \\
0\end{array}$ & $\begin{array}{c}0 \\
-2 / \sqrt{5} \\
1 / \sqrt{5} \\
0 \\
0\end{array}$ & $\begin{array}{c}-\sqrt{12} / 5 \\
0 \\
0 \\
3 / 5 \\
-2 / 5\end{array}$ & $\left.\begin{array}{c}-\sqrt{12} / 5 \\
0 \\
0 \\
-2 / 5 \\
3 / 5\end{array}\right)$ \\
\hline
\end{tabular}

Here $\tau=(\sqrt{5}+1) / 2$. 


\section{Conclusions}

In this work, the AIRs of the appropriate little groups that induce/engender the IRs of the point groups with five-fold rotational symmetry are derived by exploring the little group method in conjunction with the solvability property. It has already been established that the constants required for specifying a physical or magnetic property occurring before the degenerate IR of a chosen point group can be obtained in an elegant and simple way from the AIR of the appropriate little groups [9]. In short, the AIRs of a little group give us every information that is expected of a degenerate IR, which is induced by it, of the point group.

After the discovery of the quasicrystals [10], quite a good deal of work has been carried out on the point groups with five-fold rotational axis, for example, calculation of Raman and hyper-Raman scattering tensors, selection rules for atomic transitions, computation of spherical harmonic base for point groups with five-fold rotation axes etc. for the past two decades. The authors believe that this work would be useful for group theoretical physicists working in the area of quasicrystals in their future endeavors.

\section{References}

[1] E B Wilson, J C Decius and P C Cross, Molecular vibrations (Mc-Graw Hill, New York, 1955)

[2] F A Cotton, Chemical applications of group theory (Wiley Eastern Limited, New Delhi, 1974)

[3] C J Bradely and A P Cracknell, The mathematical theory of symmetry in solids (Clarendon Press, Oxford, 1972)

[4] J S Lomont, Application of finite groups (Academic Press, New York, 1959)

[5] C J Bradley, J. Math. Phys. 7, 1145 (1966)

[6] I V V Raghavacharyulu, Can. J. Phys. 39, 830 (1961)

[7] C H V S Rama Chandra Rao, Acta Cryst. A29, 714 (1973)

[8] S Bhagavantam and T Venkatarayudu, Theory of groups and its applications to physical problems (Academic Press, New York, 1969)

[9] T S G Krishna Murthy, L S R K Prasad and K Rama Mohana Rao, J. Math. Phys. 12, 141 (1978)

[10] D Schechtman, I Blech, D Gratias and J W Chan, Phys. Rev. Lett. 53, 1951 (1984)

[11] N B Backhouse and P Gard, J. Phys. A: Math. and Gen. 17, 2101 (1974)

[12] J Q Chen, Group representation theory for physicists (World Scientific, Singapore, 1989)

[13] L L Boyle and Y M Parker, Mol. Phys. 39, 95 (1980)

[14] L L Boyle, Int. J. Quantam Chem. 6, 919 (1972)

[15] D B Litvin, Acta Cryst. A31, 407 (1975)

[16] Fa Liu and Jia-Lun Ping, J. Math. Phys. 31(5), 1065 (1990)

[17] F Matossi, Grapeentheoric der Eigen Schwingungen Von Punktsystemen (Springer-Verlag, Berlin, 1961) 